\title{
Fatores relacionados à idade de realização do primeiro exame de mamografia em mulheres atendidas em um serviço público de Belo Horizonte - MG
}

\author{
Factors related to the age of completion of the first examination of mammography in \\ women attending in public service of Belo Horizonte - MG
}

Los factores relacionados con la edad de la realización del primer examen de mamografía en mujeres que acuden a un servicio público de Belo Horizonte - MG

Catarina Maria Nogueira de Oliveira Sediyama ${ }^{1 *}$, Manoela Maciel dos Santos Dias ${ }^{1}$, Mariana de Moura e Dias ${ }^{1}$, Milene Cristine Pessoa ${ }^{2}$, Renata Nascimento Freitas ${ }^{3}$, Sérgio Oliveira de Paula ${ }^{1}$, Maria do Carmo Gouveia Peluzio1.

\section{RESUMO}

Objetivo: Avaliar os fatores associados à realização da primeira mamografia em um serviço público de Belo Horizonte, Minas Gerais. Métodos: Foram selecionadas 378 mulheres encaminhadas para um serviço de referência. Medidas antropométricas foram aferidas e um questionário, com perguntas sobre estilo de vida, presença de comorbidades e aspectos relacionados à vida reprodutiva, foi aplicado. Resultados: A procedência urbana, o maior nível de escolaridade e renda, a pré menopausa, a ausência de terapia de reposição hormonal, de diabetes melittus e de história familiar de câncer de mama, o uso de contraceptivo oral, o sobrepeso ou obesidade e o tabagismo apresentaram associação $(p<0,05)$ com a idade mais jovem de realização da primeira mamografia. Conclusão: Sugere-se a existência de associação direta entre a realização precoce de mamografia e o maior nível de escolaridade, menopausa e obesidade. Já a procedência rural, a ausência do uso de contraceptivos orais e a eutrofia apresentaram associação inversa.

Palavras-chave: Mamografia, Epidemiologia, Câncer de mama.

\begin{abstract}
Objective: To evaluate the factors associated with completion of the first mammogram at a public hospital in Belo Horizonte, Minas Gerais, Brazil. Methods: We selected 378 women in a referral service. Anthropometric measurements were taken and a questionnaire with questions about lifestyle, presence of comorbidities and aspects related to reproductive life, was applied. Results: Urban origin, the highest level of education and income, pre menopause, the absence of hormone replacement therapy, diabetes mellitus, family history of breast cancer, oral contraceptive use, overweight or obesity and smoking were associated $(p<0.05)$ with the younger age of completion of the first mammogram. Conclusion: It is suggested that there is a direct association between early mammography and the higher level of education, menopause and obesity. However the rural areas, the absence of the use of oral contraceptives and eutrophic showed an inverse association.
\end{abstract}

Key words: Mammography, Epidemiology, Breast cancer.

\footnotetext{
1 Universidade Federal de Viçosa, Viçosa - MG. *E-mail: catynogueira@yahoo.com.br

2 Universidade Federal de Minas Gerais, Belo Horizonte - MG.

${ }^{3}$ Universidade Federal de Ouro Preto, Ouro Preto - MG.
}

SUBMETIDO EM: 5/2021

ACEITO EM: 5/2021

PUBLICADO EM: 5/2021 


\section{RESUMEN}

Objetivo: Evaluar los factores asociados a la finalización de la primera mamografía en un hospital público de Belo Horizonte, Minas Gerais, Brazil. Métodos: Se seleccionaron 378 mujeres que se refiere a un servicio de referencia. Se tomaron medidas antropométricas y se aplicó presencia de comorbilidades y los aspectos relacionados con la vida reproductiva de un cuestionario con preguntas sobre el estilo de vida. Resultados: Origen urbano, el más alto nivel de educación y los ingresos, la menopausia antes, la ausencia de terapia de reemplazo hormonal, diabetes mellitus, historia familiar de cáncer de mama, el uso de anticonceptivos orales, el sobrepeso o la obesidad y cigarrillos se asociaron $(\mathrm{p}<0,05)$ con la edad más joven de la finalización de la primera mamografía. Conclusión: Se sugiere que existe una relación directa entre la mamografía temprana y el mayor nivel de educación, la menopausia y la obesidad. Ya las zonas rurales, la ausencia del uso de anticonceptivos orales y eutróficos mostraron una asociación inversa.

Palabras clave: Mamografía, Epidemiología, Cáncer de mama.

\section{INTRODUÇÃO}

Segundo a Organização Mundial de Saúde, a detecção precoce continua a ser a pedra angular de controle do câncer de mama (WHO, 2021). Contudo, apesar dos avanços no conhecimento científico e dos aparatos utilizados nas intervenções, ainda hoje, o diagnóstico é tardio, com mortalidade elevada em todo o mundo, sendo o câncer de mama, a quinta causa de óbito por câncer no mundo (FERLAY J, et al., 2015). A estimativa brasileira de novos casos de câncer de mama para o ano de 2020 é de 70.000, com mortalidade crescente no Brasil nas últimas décadas, chegando a 12,66 mulheres a cada 100.000 habitantes (INCA 2019; INCA 2016).

Existe no país um programa de rastreamento mamográfico nacional, que atua na prevenção secundária da população assintomática, objetivando o diagnóstico precoce e a redução da mortalidade (INCA 2021). Contudo, destaca-se que, no Brasil, fatores como baixa renda, idade avançada e grupo étnico podem contribuir para a pouca aderência a realização rotineira do exame de mamografia (AMORIM VMSL, et al., 2008).

A mamografia é o método mais efetivo para o diagnóstico precoce da doença, além de ser o único exame de rastreamento com impacto comprovado na redução da mortalidade pelo câncer de mama (WHO, 2021; INCA 2017). A redução de risco de desenvolver câncer de mama com o rastreamento mamográfico em geral chega a $19 \%$, variando desde $15 \%$ naquelas mulheres na faixa dos 40 anos até $32 \%$ entre aquelas na faixa dos 60 anos, com um risco cumulativo de exame falso positivo de $61 \%$ para as mulheres entre 40 e 50 anos em rastreamento mamográfico anual (PACE L e KEATING N, 2014).

As mulheres com risco médio para a doença, devem iniciar o rastreamento aos 45 anos de maneira regular (OEFFINGER K, et al., 2015). Além disso, mulheres com risco aumentado para o desenvolvimento do câncer de mama, ou seja, aquelas com presença de caso da doença em parente de primeiro grau (unilateral) com idade inferior a 50 anos, mulheres com presença de história familiar de câncer de mama masculino, de câncer de mama bilateral ou de ovário e pacientes com história prévia de lesão mamária proliferativa com atipia ou neoplasia lobular in situ devem iniciar o rastreamento aos 35 anos (INCA 2021).

Sendo a idade um fator de risco conhecido para o câncer de mama e muitas vezes o único fator de risco identificado, em mulheres com idade acima dos 70 anos a mamografia deverá se estender bianualmente, quando houver boas condições de saúde (WHO 2021; PINHO VFDS; COUTINHO ESF, 2007; OEFFINGER $\mathrm{K}$, et al., 2015). De acordo com Howlader N, et al. (2012), o risco de desenvolver câncer de mama entre mulheres americanas é crescente com a idade, chegando a 3,82\% aos 70 anos.

Com base no exposto, esse estudo tem como objetivo analisar os fatores associados à idade de realização da primeira mamografia em mulheres atendidas pelo Sistema Único de Saúde (SUS) na cidade de Belo Horizonte, Minas Gerais, Brasil. 


\section{MÉTODOS}

Esse estudo foi realizado com mulheres encaminhadas para a realização de mamografia, em um serviço de mastologia, na cidade de Belo Horizonte, MG, entre janeiro e julho de 2006.

O estudo seguiu a Declaração de Helsinki e foi aprovada pelo Comitê Nacional de Ética em Pesquisa do Conselho Nacional de Saúde (CONEP/CNS/MS), com parecer número 1889/2005.

Mulheres com idade inferior a 18 anos, que nunca realizaram exames de mamografia, ou que não se recordavam a idade de realização do primeiro exame foram excluídas do estudo. Assim, 378 mulheres foram incluídas e aceitaram participar do estudo, através da assinatura do Termo de Consentimento Livre e Esclarecido.

A coleta de dados foi realizada no ambulatório de um serviço de mastologia, utilizando-se questionário previamente validado (ABRANCHES MV, et al., 2011; PENA GG, et al., 2013).

$\mathrm{Na}$ entrevista foram coletadas informações socioeconômicas como procedência, situação conjugal, grau de escolaridade, tipo de ocupação, renda líquida familiar mensal (aproximada e per capita) e número de membros da família, bem como informações clínicas como presença de diabetes (glicemia $>126 \mathrm{mg} / \mathrm{dL}$ ), gota ou insuficiência renal, história prévia de doença benigna e história familiar de câncer de mama.

Sobre as variáveis relacionadas à história ginecológica da mulher, questionou-se sobre a data da menarca, a idade da menopausa, o número de filhos, a presença de aborto, a realização de amamentação, o tempo de amamentação de cada filho, a idade da primeira gestação, o uso de contraceptivo oral e o uso de terapia de reposição hormonal.

Em relação a avaliação antropométrica aferiu-se o peso (balança eletrônica TANITA®) e altura (antropômetro vertical Alturaexata®) das voluntárias. Com os dados resultantes da aferição, calculou-se o índice de massa corporal (IMC) das mulheres. Foram consideradas eutróficas as mulheres com IMC entre 18,5 a 24,9 kg/m2; com sobrepeso, as mulheres com IMC entre 25,0 a 29,9 kg/m2 e, como obesas, as mulheres com IMC maior que $30,0 \mathrm{~kg} / \mathrm{m} 2$ (WHO, 1995).

Quanto à circunferência da cintura, essa medida foi aferida com o auxílio de fita inelástica, sendo que se utilizou como ponto de corte $80 \mathrm{~cm}$, que é indicativo de risco para o desenvolvimento de co-morbidades associadas (WHO, 2004a). Por fim, para a determinação do percentual de gordura corporal, utilizou-se a bioimpedância elétrica vertical TANITA® (Modelo TBF $531 \AA$, Tanita Corporation of America, Illinois, USA), com precisão de 1\% para gordura corporal, sendo a classificação do estado nutricional, segundo Gallagher D, et al. (2000). Questionamentos sobre peso habitual, peso aos 18 anos e ganho ou perda de peso desde os 18 anos também foram realizadas

Em relação ao estilo de vida das pacientes, avaliou-se o tabagismo, considerando-se tabagistas as voluntárias que fumavam ao menos 1 cigarro por dia, independentemente de a quantos anos essa prática era realizada; o etilismo, avaliando-se o consumo de álcool de acordo com WHO (2004b); e a prática de atividade física, através de Questionário Internacional de Atividade Física (IPAQ), versão curta (CELAFISCS, 2004).

A análise dos dados foi realizada utilizando-se o programa Stata, versão 9.1. Para cada variável foi testada a normalidade pelo teste de Kolmogorov-Smirnov. Para análises comparativas, as variáveis com distribuição normal foram avaliadas com o teste t Student, e aquelas de distribuição não normal foram avaliadas pelo teste Mann-Whitney, para comparação entre 2 amostras, ou Kruskal-Wallis, para comparação entre 3 ou mais amostras.

A correlação entre o desfecho (idade de realização da primeira mamografia) e cada variável categórica foi avaliada usando análises univariadas (qui-quadrado e odds ratio bruta) e a análise multivariada (regressão logística). As variáveis que, na análise univariada, mostraram-se associadas com a idade de realização da primeira mamografia, com nível de significância observado menor ou igual a $20 \%$ no teste do qui-quadrado $(p \leq 0,20)$, foram selecionadas para a análise multivariada por meio de regressão logística. $O$ nível de significância considerado foi de $5 \%(p<0,05)$. 


\section{RESULTADOS}

Foram avaliadas 378 mulheres, com mediana de idade de 51 anos. Dessas, 40,06\% eram provenientes da zona rural, $21,16 \%$ eram solteiras e $29,90 \%$ tinham sete ou mais anos de estudo completos. A história pregressa de doença benigna da mama foi descrito por $9,25 \%$ das voluntárias e a história familiar de câncer de mama por $16,66 \%$ das mulheres. Nuliparidade estava presente em $15,88 \%$ e o aleitamento materno foi relatado por $78,31 \%$ das pacientes. A Terapia de Reposição Hormonal (TRH) foi ou era realizada por $16,40 \%$ das entrevistadas (Tabela 1).

Tabela 1 - Características sociodemográficas, antropométricas e relacionadas à vida sexual e ao estilo de vida de mulheres atendidas em um setor de mamografia de Belo Horizonte - MG.

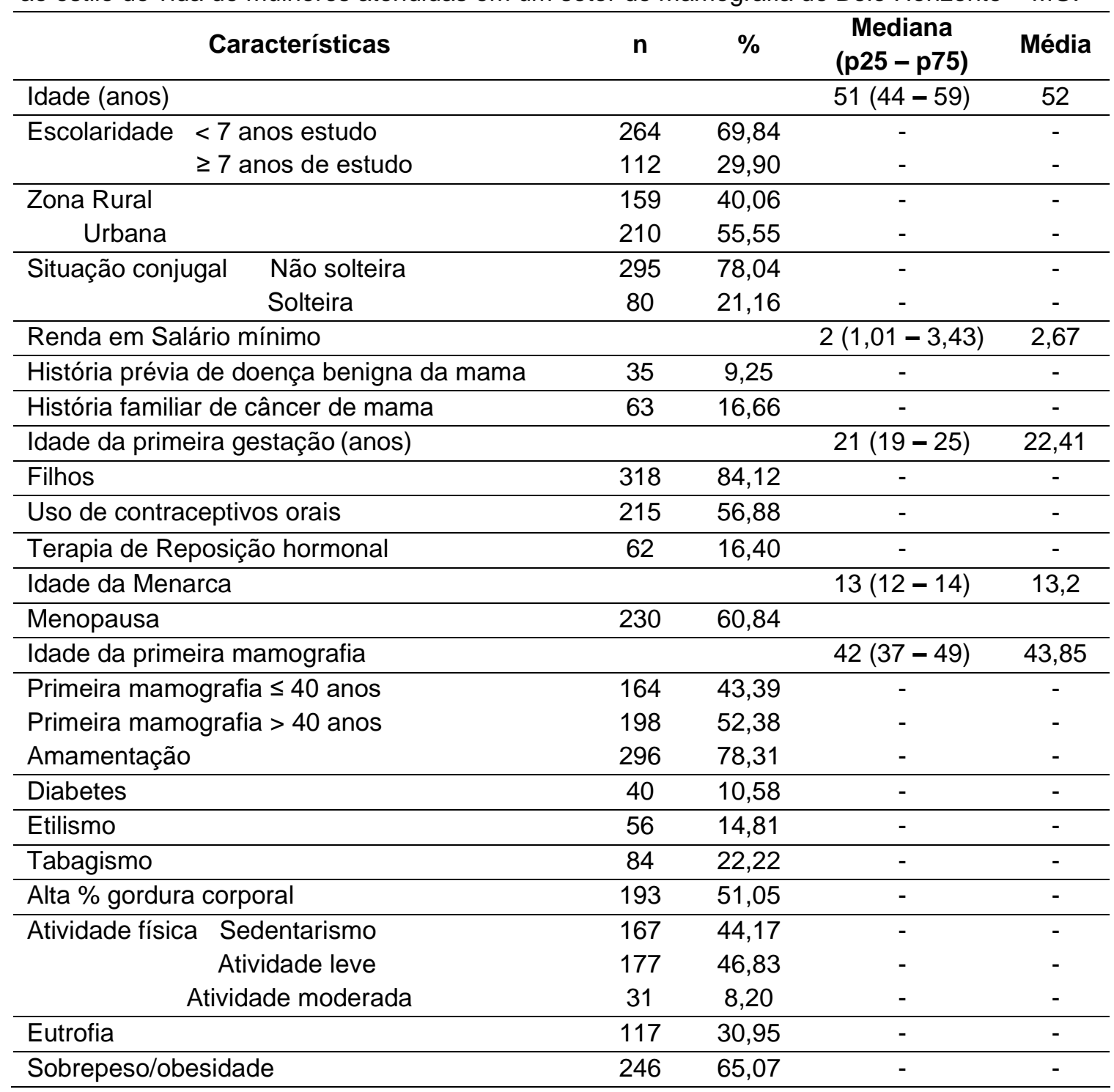

Nota: Resultados expressos em valores absolutos e percentuais.

Fonte: Sediyama CMNO, et al., 2021.

A idade de realização da primeira mamografia foi menor entre as mulheres que residiam na área urbana em relação aquelas da área rural; naquelas com maior escolaridade; com maior renda; pré-menopausadas; que não realizaram terapia de reposição hormonal; que usavam ou relataram uso de contraceptivos orais; e entre as tabagistas. Observou-se também que a idade de realização da primeira mamografia foi maior entre as pacientes portadoras de diabetes melittus e com história familiar de câncer de mama, quando comparadas aquelas sem essa característica (Tabela 2). 
Tabela 2 - Idade de realização da primeira mamografia (anos) de acordo com as características avaliadas em mulheres atendidas em um setor de mamografia de Belo Horizonte - MG

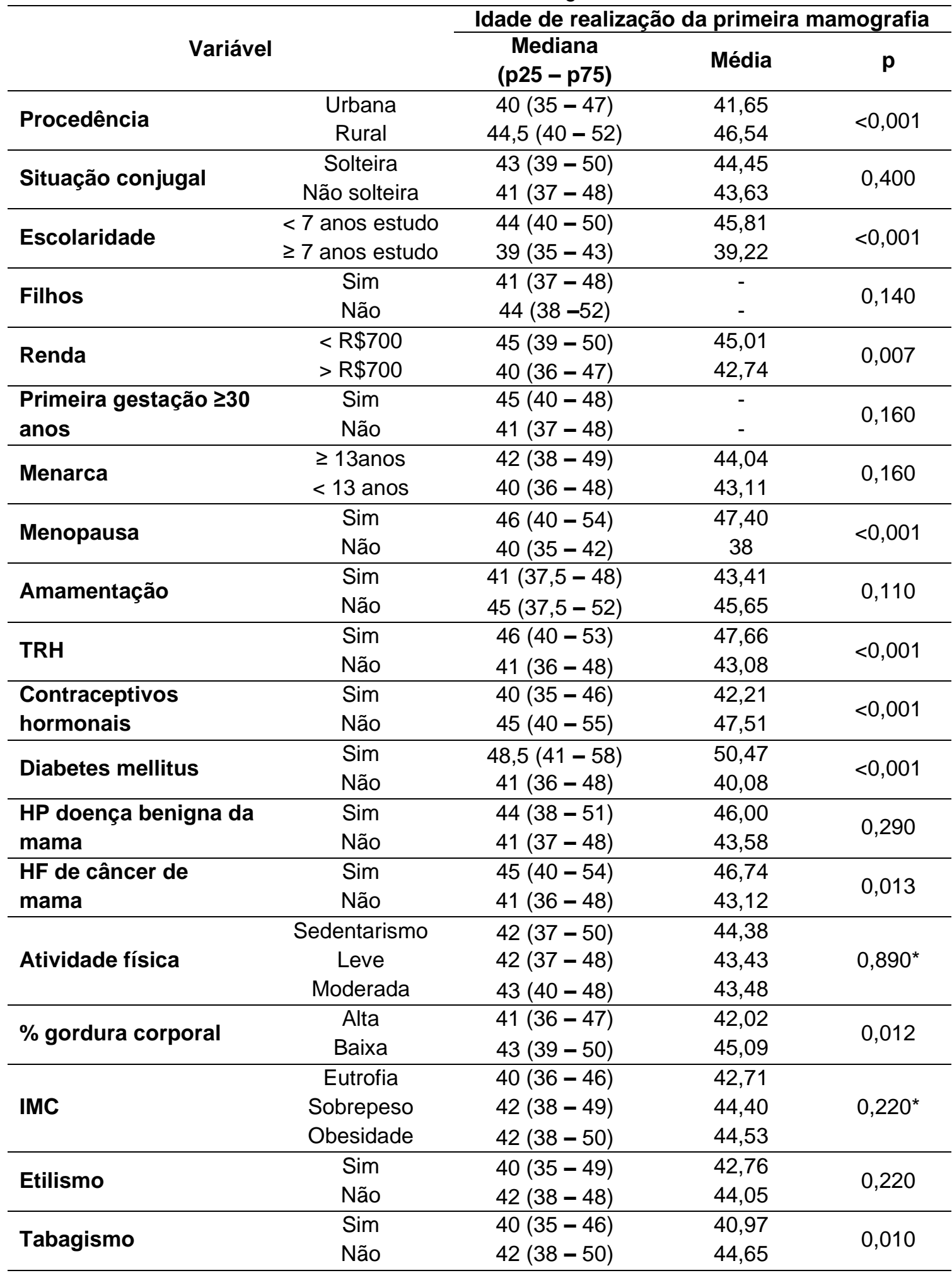

Nota: Resultados expressos em valores absolutos. Teste de Mann Whitney, para comparação entre 2 amostras, ou teste Kruskal Wallis $\left(^{*}\right)$, para comparação entre 3 amostras. Dados com distribuição não paramétrica. Onde: $\mathrm{TRH}=$ terapia de reposição hormonal; $\mathrm{HP}=$ história pregressa; $\mathrm{HF}=$ história familiar; IMC = índice de massa corporal.

Fonte: Sediyama CMNO, et al., 2021. 
Na Tabela 3 pode-se verificar a associação direta da realização precoce da primeira mamografia com a obesidade, o maior grau de escolaridade e a menopausa, e a associação inversa foi observada para a procedência da zona rural, o baixo percentual de gordura corporal, e a ausência do uso de contraceptivos orais.

Tabela 3 - Características relacionadas à idade de realização da primeira mamografia em mulheres atendidas em um setor de mamografia de Belo Horizonte - MG

\begin{tabular}{|c|c|c|c|}
\hline Variável & $O R$ & $95 \%$ IC & $\mathbf{p}$ \\
\hline Menopausa & 2,80 & $1,62-4,87$ & $<0,001$ \\
\hline Zona rural & 0,50 & $0,29-0,87$ & 0,013 \\
\hline Escolaridade maior & 2,55 & $1,35-4,83$ & 0,004 \\
\hline Não usar contraceptivo oral & 0,56 & $0,32-0,98$ & 0,041 \\
\hline Baixo \% gordura corporal & 0,41 & $0,20-0,87$ & 0,020 \\
\hline & 2,07 & $0,96-4,44$ & 0,062 \\
\hline Obesidade & 3,41 & $1,33-8,79$ & 0,011 \\
\hline
\end{tabular}

Nota: Resultados expressos em valores absolutos. Teste de qui-quadrado. Onde: OR $=$ odds ratio; $\mathrm{Cl}=$ intervalo de confiança; IMC = índice de massa corporal.

Fonte: Sediyama CMNO, et al., 2021.

\section{DISCUSSÃO}

O rastreamento assintomático de mulheres entre 40 e 49 anos foi avaliado em um serviço público no Brasil, encontrando baixa frequência de diagnóstico de câncer de mama (SILVA FX, et al., 2014). No presente estudo, 164 mulheres realizaram a primeira mamografia com idade inferior a 40 anos. Fatores como tabagismo, renda, escolaridade, presença de comorbidade, procedência e fatores relacionados ao ciclo menstrual dessas mulheres estavam associados à realização precoce do exame.

A população avaliada realizou a primeira mamografia com idade inferior ao preconizado para início de rastreamento mamográfico pelo Ministério da Saúde (MS) (BRASIL 2004; BRASIL 2013). O MS recomenda o exame clínico anual das mamas a partir dos 40 anos, e em casos alterados, mamografia. $O$ rastreamento mamográfico bilateral deverá ser realizado a partir dos 50 anos, com intervalo máximo de dois anos. Caso a mulher pertença a algum grupo de risco elevado para essa doença, o exame clínico da mama e a mamografia unilateral devem ser realizados anualmente a partir dos 35 anos (INCA 2021; BRASIL 2013).

Pereira MB, et al. (2014), em revisão sobre a idade de realização e periodicidade da mamografia, sugeriu que o exame tenha regularidade bianual entre mulheres com idade entre 50 e 69 anos (PEREIRA MB, et al., 2014). Sobre a idade inferior e superior, mais estudos são necessários para definição da periodicidade da realização do exame. Contudo, no Brasil, a Sociedade Brasileira de Mastologia ainda recomenda a realização do rastreamento mamográfico bilateral bianual a partir dos 40 anos (SBM 2017).

Corroborando com as recomendações do MS, alguns países também não recomendam a realização rotineiramente da mamografia a partir dos 40 anos, devendo a decisão do rastreamento bianual em mulheres entre 40-49 anos ser individualizada. Para aquelas com idade entre 50 e 74 anos, essa avaliação pode ser realizada a cada dois anos (US PREVENTIVE SERVICES TASK FORCE 2009; KALAGER M, et al., 2010). Enquanto que nos Estados Unidos, a recomendação é de mamografia anual dos 45 aos 54 anos, e bianual a partir dos 55 anos. Mulheres entre 40 e 44 anos poderão ser rastreadas anualmente (OEFFINGER K, et al., 2015).

As pacientes provenientes da área urbana da cidade de Belo Horizonte, MG realizaram o exame com idade inferior aquelas da zona rural. $O$ acesso a serviços de saúde e o acesso ao exame com maior facilidade para aquelas da região metropolitana de Belo Horizonte, MG pode ser uma possível explicação para essa diferença encontrada de acordo com a procedência das mulheres. A associação inversa entre a procedência rural e a realização da mamografia precocemente se manteve presente no modelo estatístico final. 
Mulheres com maior grau/ nível de escolaridade realizaram a primeira mamografia com idade inferior ao grupo com menos anos de estudo, sendo que o grau de entendimento sobre a doença e o exame podem ser fatores determinantes na adesão à rotina de exames das mamas. Observou-se que um terço das entrevistadas usuárias do serviço de saúde pública não realizaram a mamografia conforme solicitação médica (GODINHO ER e KOCH HA, 2002). Além disso, a não realização da mamografia e do exame clínico das mamas também foi associada ao baixo grau de escolaridade entre mulheres na cidade de Campinas, SP (AMORIM VMSL, et al., 2008).

As mulheres de maior renda realizaram a primeira mamografia com idade inferior àquelas com menor renda. Godinho ER e Koch HA (2002) descreveram que 10\% das mulheres no serviço público, avaliadas em um estudo na cidade de Goiânia, GO, não realizaram a mamografia por questões financeiras (GODINHO ER e KOCH HA, 2002). Amorim VMSL, et al. (2008) em um estudo na cidade de Campinas, SP, encontraram uma menor realização do exame na faixa de menor nível socioeconômico, inferindo ainda que a não realização de mamografia e do exame clínico das mamas estava relacionado ao fato de pertencer ao segmento de menor renda familiar per capita (AMORIM VMSL, et al., 2008). Em ambos os estudos não foi avaliada a idade de realização do exame de acordo com a condição econômica dessas mulheres.

Em um estudo avaliando os fatores associados à realização de mamografia por mulheres brasileiras, encontrou-se uma prevalência de $36 \%$ de mamografia e dentre os fatores preditivos para a realização do exame estava a renda elevada, a faixa etária entre 40 e 59 anos, o acesso a planos de saúde e a realização de consulta médica no último ano (NOVAES HMD, et al., 2006). Ainda, em um estudo na cidade de Guarapuava, PR, a realização de exame preventivo de mama foi influenciada pela baixa renda e baixa escolaridade, ou seja, quanto menor o nível de renda e de formação, menor é o acesso ao exame de mamografia (BIM CR, et al., 2010).

Observou-se que 16,66\% das mulheres possuíam história familiar de câncer de mama em pelo menos um parente de primeiro grau. Visto que o histórico de doença benigna na mama é um fator de risco para o câncer de mama (INCA 2021) rastreamento precoce é indicado nesses casos. A idade de realização da primeira mamografia nessas mulheres foi superior aquelas sem história familiar $(p=0,013)$. Estudo de Godinho ER e Koch HA (2002) encontrou relato de história familiar de câncer de mama em 13,3\% da população avaliada que foi atendida em um hospital público. Dessas $28,7 \%$ não tinham realizado mamografia prévia.

As mulheres com idade entre 40 e 49 anos com risco duas vezes maior de câncer de mama podem se beneficiar do mesmo rastreamento realizado para aquelas com idade acima de 50 anos (VAN RAVESTEYN NT, et al., 2012), podendo o rastreamento ser direcionado àquelas com características que possam conferir aumento de risco para câncer mama, como a história familiar de câncer de mama, a alta densidade do tecido mamário e a história de doença benigna da mama (KERLIKOWSKE K, 2012).

As mulheres que relataram uso de terapia de reposição hormonal (TRH) realizaram a primeira mamografia com idade superior ao grupo que não fazia uso dessa terapia. Entre as mulheres que faziam TRH, $22,5 \%$ tinham história de câncer de mama na família. Este é um conhecido fator de risco para o câncer de mama, devendo as mulheres nessa condição estar sob vigilância precoce (AMORIM VMSL, et al. 2008). Em estudo realizado na cidade de Goiânia, GO, entre as mulheres que se submeteram a mamografia, $20 \%$ utilizavam $\mathrm{TRH}$, sendo que um quinto destas relataram não ter realizado exame mamográfico prévio (GODINHO ER e $\mathrm{KOCH} \mathrm{HA,} \mathrm{2002).} \mathrm{Esse} \mathrm{é} \mathrm{um} \mathrm{dado} \mathrm{preocupante} \mathrm{uma} \mathrm{vez} \mathrm{que} \mathrm{o} \mathrm{início} \mathrm{tardio} \mathrm{de} \mathrm{rastreamento} \mathrm{poderá} \mathrm{prejudicar}$ a eficiência do rastreamento nessa população.

O uso de contraceptivos orais é outra condição associada ao câncer de mama (AMERICAN CANCER SOCIETY AC, 2019). No presente estudo as mulheres que não relataram uso de anticoncepcionais, no momento da pesquisa ou no passado, realizaram a primeira mamografia com idade superior aquelas que relataram essa condição. A não utilização de contraceptivos orais foi associada indiretamente a idade de realização da primeira mamografia no modelo estatístico final. Em um estudo que avaliou a realização de mamografia em mulheres assintomáticas entre 40 e 49 anos, 6,8\% delas faziam uso de anticoncepcionais orais (SILVA FX, et al., 2014). No presente estudo $57,64 \%$ das mulheres relataram uso desses medicamentos em momento atual ou pregresso. 
Freitas Júnior $F$, et al. (2006), relacionaram o uso de anticoncepcionais orais à dor e ao desconforto durante a realização do exame, possivelmente por maior sensibilidade mamária durante o uso do medicamento, fato esse que poderia influenciar a não realização do exame no futuro em $25 \%$ dos casos.

O risco de câncer de mama aumenta com a idade, com o uso de TRH, com o sobrepeso e a obesidade e entre as mulheres que entraram na menopausa com idade acima de 55 anos (AMERICAN CANCER SOCIETY AC, 2019). Logo, considerando que um quinto das mulheres faziam uso de reposicão hormonal e que um quarto tinham história familiar de câncer de mama, a idade de realização do inicio do rastreamento deveria ter sido menor. Também, a realização precoce do rastreamento mamografico entre mulheres com sobrepeso e obesidade deveria ter sido mais precoce, o que não foi observado em nosso estudo.

Mulheres não diabéticas realizaram a primeira mamografia mais precocemente do que as com a doença. Estudo em Terezina, PI, mostrou que apenas $40 \%$ das diabéticas realizaram mamografia nos 2 anos anteriores, sendo essa ação preventiva pouco realizada devido a baixa escolaridade e a não realização do controle glicêmico, o que prejudica a aderência ao rastreamento do câncer de mama (REZENDE NETA D, et al., 2011). Isso é grave visto que níveis glicêmicos elevados podem influenciar negativamente o prognóstico de mulheres portadoras de câncer de mama (MONZAVI-KARBASSI B, et al., 2016).

Por fim, a idade de realização da mamografia foi menor entre as tabagistas, sugerindo-se uma preocupação em relação as consequencias do tabagismo no aumento da incidência de câncer. Logo, é crescente o desafio dos gestores em garantir o acesso ao exame de mamografia pela população geral, especialmente pelos mais vulnerávéis. Acredita-se que atenção especial é necessaria na implantação de estratégias visando a estratificação do risco de câncer de mama e a correta indicação do início do rastreamento mamográfico, a fim de garantir a adoção de práticas preventivas direcionada a essa população.

\section{CONCLUSÃO}

O presente estudo sugere a existência de associação direta entre a realização precoce de mamografia e o maior nível de escolaridade, menopausa e obesidade. A procedência rural, a ausência do uso de contraceptivos orais, e a eutrofia apresentaram associação inversa no modelo estatístico final. Além disso, a realização de mamografia antes dos 50 anos é direcionada pela existência de riscos para o câncer de mama, mas deveria também, levar em conta questões relacionadas à presença de comorbidades e estilo de vida das pacientes. Logo, é preciso estar atento aos fatores que contribuem negativamente para a realização tardia do primeiro exame de mamografia, além de identificar precisamente a população de maior risco de câncer de mama para uma abordagem direcionada do programa de rastreamento mamográfico em relação a idade de início da realização do exame e sua correta indicação.

\section{REFERÊNCIAS}

1. ABRANCHES MV, et al. Antioxidant vitamins and cytokines are altered in breast cancer. European Journal of Cancer Prevention, 2011; 20 (5): 403-410.

2. AMERICAN CANCER SOCIETY . Breast Cancer. Facts \& Figures. 2019. Disponível em: https://www.cancer.org/content/dam/cancer-org/research/cancer-facts-and-statistics/breast-cancer-facts-andfigures/breast-cancer-facts-and-figures-2019-2020.pdf. Acesso em: 26 de abril de 2021.

3. AMORIM VMSL, et al. Fatores associados a não realização da mamografia e do exame clínico das mamas: um estudo de base populacional em Campinas, São Paulo, Brasil. Cadernos de Saúde Pública, 2008; 24 (11): 2623-2632.

4. BIM CR, et al. Diagnóstico precoce do câncer de mama e colo uterino em mulheres do município de Guarapuava, PR, Brasil. Revista da Escola de Enfermagem da USP, 2010; 44(4): 940-946.

1. 28

5. BRASIL. Ministério da Saúde. Controle do câncer de mama - Documento de consenso. Revista Brasileira de Cancerologia, 2004; 50(2): 77-90.

6. BRASIL. Ministério da Saúde. Portaria nํ 1.253, de 12 de novembro de 2013. Brasília: Ministério da Saúde, 2013. Disponível em: http://bvsms.saude.gov.br/bvs/saudelegis/sas/2013/prt1253_12_11_2013.html. Acessado em: 26 de abril de 2021.

7. CELAFISCS. Centro de Estudos do Laboratório de Aptidão Física São Caetano do Sul. International Physical Activity Quationnaire - Short Version. São Caetano do Sul, 2004. 
2. 8.FERLAY J, et al. Cancer incidence and mortality worldwide: Sources, methods and major patterns in GLOBOCAN 2012. International Journal of Cancer, 2015; 136 (5): E359-E386.

8. FREITAS JÚNIOR R, et al. Desconforto e dor durante realização da mamografia. Revista da Associação Médica Brasileira, 2006; 52(5): 333-336.

9. GALLAGHER D, et al. Health percentage body fat ranges: an approach for developing guidelines based on body mass index. American Journal of Clinical Nutrition, 2000; 72: 694-701.

10. GODINHO ER, KOCH HA. O perfil da mulher que se submete a mamografia em Goiânia: uma contribuição a "Bases para um programa de detecção precoce do câncer de mama". Radiologia Brasileira, 2002; 35(3): 139-145.

11. HOWLADER N, et al. SEER Cancer Statistics Review, 1975-2009. Bethesda, MD: National Cancer Institute, 2012.

12. INSTITUTO NACIONAL DO CÂNCER (INCA). Ministério da Saúde. Atlas On-line de Mortalidade. 2016. Disponível em: https://mortalidade.inca.gov.br/MortalidadeWeb/. Acessado em: 26 de abril de 2021.

13. INSTITUTO NACIONAL DO CÂNCER (INCA). Ministério da Saúde. Diretrizes para a detecção precoce do câncer de mama no Brasil. Sumário executivoo. 2017.2 Disponível em: https://www.inca.gov.br/sites/ufu.sti.inca.local/files//media/document//sumario-diretrizes-deteccao-precoce-mama2017.pdf. Acessado em: 26 de abril de 2021.

14. INSTITUTO NACIONAL DO CÂNCER (INCA). Ministério da Saúde. Estimativa 2020: Incidência no câncer no Brasil. 2019. Disponível em: https://www.inca.gov.br/publicacoes/livros/estimativa-2020-incidencia-de-cancer-no-brasil. Acessado em: 07 de abril de 2021.

15. INSTITUTO NACIONAL DO CÂNCER (INCA). Ministério da Saúde. Parâmetros técnicos para o rastreamento do câncer de mama. Recomendações para gestores estaduais e municipais. Rio de Janeiro: Ministério da Saúde, 2021. Disponível

em: https://www.inca.gov.br/sites/ufu.sti.inca.local/files//media/document/parametrostecrastreamentocamama_2021_0.p df . Acessado em: 26 de abril de 2021.

16. KALAGER M, et al. Effect of screening mammography on breast cancer mortality in Norway. New England Journal of Medicine, 2010; 363(13): 1203-1210.

17. KERLIKOWSKE K. Screening mammography in women less than age 50 years. Current Opinion in Obstetrics and Gynecology, 2012; 24 (1): 38-43.

18. MONZAVI-KARBASSI B, et al. Pre-diagnosis blood glucose and prognosis in women with breast cancer. Cancer \& Metabolism, 2016; 4(7).

19. NOVAES HMD, et al. Fatores associados à realização de exames preventivos para câncer nas mulheres brasileiras, PNAD 2003. Ciência e Saúde Coletiva, 2006; 11(4): 1023-1035.

20. OEFFINGER K, et al. Breast cancer screening for women at average risk: Guideline update from the American Cancer Society. JAMA, 2015; 314 (15): 1599-1614.

21. PACE L, KEATING N. A systematic assessment of benefits and risks to guide breast cancer screening decisions. JAMA, 2014; 311 (13): 1327-1335.

22. PENA GG, et al. Physical activity is associated with malignant and benign breast diseases in low-income brazilian women. Nutrition and Cancer, 2013; 66 (4): 707-715.

23. PEREIRA MB, et al. Grupo etário e periodicidade recomendados para a mamografia de rastreio: uma revisão sistemática. Ciência e Saúde Coletiva, 2014; 19(4): 1135-1140.

24. PINHO VFS, COUTINHO ESF. Variáveis associadas ao câncer de mama em usuárias de unidades básicas de saúde. Cadernos de Saúde Publica, 2007; 23 (5): 1061-1069.

25. REZENDE NETA D, et al. Prevention of breast cancer in women with diabetes mellitus. Northeast Network Nursing Journal, 2011; 12 937-42.

26. SCREENING FOR BREAST CANCER US. Preventive services task force. Recommendation statement. Annals of Internal Medicine 2009; 151(10): 716-726.

27. SOCIEDADE BRASILEIRA DE MASTOLOGIA (SBM). Sociedades brasileiras recomendam mamografia a partir dos 40 anos. 2017. Disponível em: https://www.sbmastologia.com.br/noticias/sociedades-medicas-brasileirasrecomendam-mamografia-anual-a-partir-dos-40-anos/. Acessado em: 26 de abril de 2021.

28. SILVA FX, et al. Mamografia em mulheres assintomáticas na faixa etária de 40 a 49 anos. Revista de Saúde Pública, 2014; 48(6): 931-939.

29. VAN RAVESTEYN NT, et al. Tipping the balance of benefits and harms to favor screening mammography starting at age 40 years: a comparative modeling study of risk. Annals of Internal Medicine, 2012; 156(9): 609-617.

30. WORLD HEALTH ORGANIZATION (WHO). Physical Status: the use and the interpretation of antropometry. Geneva, 1995, 452p. Disponível em: https://www.who.int/childgrowth/publications/physical_status/en/. Acessado em: 26 de abril de 2021.

31. WORLD HEALTH ORGANIZATION (WHO). Diet, Nutrition and the prevention of chronic diseases. Geneva, 2004a, 148p. Disponível em: https://www.who.int/dietphysicalactivity/publications/trs916/en/. Acessado em: 26 de abril de 2021.

32. WORLD HEALTH ORGANIZATION (WHO). Global Status report on alcohol 2004. Geneva, 2004b, 88p. Disponível em: https://www.who.int/substance_abuse/publications/globalstatusreportalcoholchapters/en/. Acessado em: 26 de abril de 2021.

33. WORLD HEALTH ORGANIZATION (WHO). Breast CANCER: prevention and control. 2021. Disponível em: https://www.who.int/cancer/detection/breastcancer/en/. Acessado em: 26 de abril de 2021. 